\title{
Analytic Study of Doppler-based Handover Management in LEO Satellite Systems
}

\author{
Evangelos Papapetrou, Member, IEEE, and Fotini-Niovi Pavlidou, Senior, IEEE
}

\begin{abstract}
The problem of dynamic handover management in Low Earth Orbit (LEO) satellite systems is addressed in this paper. Particularly, an analytical study of a newly proposed method for handover management, called Dynamic Doppler-based Handover Prioritization scheme (DDBHP), will be presented. DDBHP utilizes Doppler shift monitoring of each communicating user terminal onboard the satellite and geometric characteristics to accurately predict the handover load. As a result, handover requests are more effectively managed, resulting to an improved performance in terms of blocking and forced termination probabilities. Moreover, by supporting guaranteed handovers, DDBHP can be used to provide QoS to users of future broadband satellite networks. An extensive mathematical model that justifies Doppler shift monitoring will be presented along with a detailed queueing model used not only to evaluate DDBHP performance but also to provide a methodology for associating DDBHP operational parameters with desired performance. Comparison of analytical and simulation results validate the proposed model.
\end{abstract}

Index Terms-satellites, handover, low earth orbit, QoS, satellite fixed cells

\section{INTRODUCTION}

$\mathbf{S}$ UPPORTING real-time and interactive services in a LEO satellite environment is a difficult task encumbered not only by physical medium characteristics such as signal quality, propagation delay, e.t.c., but also by system characteristics like the asynchronous rotation of Earth and low orbit satellites. In such a context it may be required for the system to switch a user between different satellites (handover). Although satellite systems are designed as to provide at least one visible satellite at each location, resources in each satellite do not exist a priori. Consequently a forced termination of the user-satellite connection (Up-Down Link - UDL) may occur, depending on the mechanism used to carry out handovers. The more frequent a satellite serving a region changes, the more difficult is for a satellite system to achieve the continuous serving of users. The situation is aggravated by the fact that in order to increase frequency reuse many satellite systems divide satellite footprint into cells. Due to the relatively small size of cells a user with a call in progress will need to switch from a cell to another more frequently. In fact there are two types of handovers, the satellite and the beam handover. While the former refers to the switching of a user from a satellite to another, the latter refers to the switching between cells.

Techniques that aim at eliminating interrupts in the UDL operation, thus allowing unhampered communication, are of

E. Papapetrou is with the Department of Computer Science, University of Ioannina, Ioannina, 45110, Greece (email:epap@cs.uoi.gr)

F.-N. Pavlidou is with the Department of Electrical \& Computer Engineering, Aristotle University of Thessaloniki, Thessaloniki, 54124, Greece (email:niovi@eng.auth.gr) special interest especially in the context of modern communication systems which point at providing seamlessly high quality services by utilizing a satellite component able to support such service demands. Low Earth Orbit (LEO) satellite systems [1] are the most convenient solution because they provide low propagation delays. Although this is an essential advantage for real-time and interactive services, handovers may reduce the quality of service delivered to the users. Examples of service degradation, as experienced by the user point of view, are forced termination of calls, queueing delay, e.t.c. On the other hand from the system point of view, performance degradation is related to inefficient utilization of resources.

Various studies have addressed the issue of handover management. One proposed approach is to handle handover upon its occurrence. Queuing of handovers [2]-[3] is foreseen if available resources are not present. This technique avoids protracted reservation of resources and favors low blocking probability. Nevertheless it introduces delay and relatively high forced termination probability if the acceptable queueing delay is low. In [2] E. Del Re et al proposed a handover prioritization scheme for different channel allocation techniques. This scheme proposes the queuing of handover requests $(\mathrm{QH})$ for a maximum time interval in case there is no channel available in the destination cell. The call will be forced into termination if no channel is made available within the defined time limit. Additionally in [3] different queuing policies are studied for the $\mathrm{QH}$ method.

A second approach for managing handovers is to reserve resources before handover occurrence in order to minimize forced termination probability. The reservation may be predetermined (guard channels-[4],[5]) or based on a prediction of handover requests [6]-[7]. Especially in [7] handover management is considered as part of an end-to-end routing protocol. It takes into account traffic density in a cell to predict the number of handovers and reserve channels. In this case although no delay is imposed, a cautious planning is needed to avoid an undesirable increase of blocking probability. In [6] G. Maral et al proposed a guaranteed handover service $(\mathrm{GH})$ in systems where channels are fixed allocated to cells. According to the proposed method calls requesting the guaranteed handover service are admitted in the network only if a free channel exists both in the serving and in the next cell. When the first handover occurs, a channel is requested from the following cell and so on. If the latter request can not be satisfied, it is queued until the actual handover occurrence.

The authors proposed in [8] a new method for managing handovers, called Dynamic Doppler-based Handover Prioriti- 
zation Scheme (DDBHP). DDBHP is designed for systems in which cells are fixed to satellites and aims at providing users with different levels of quality of service in terms of forced termination probability and at the same time utilize efficiently network resources. The new algorithm can also guarantee handovers, a property very useful for future satellite networks. The proposed technique takes advantage of the Doppler effect to derive the location of mobile stations (MS's) and therefore make resource reservations in due course, maximizing channel utilization and bandwidth efficiency. DDBHP uses a deterministic rather than a predictive approach for estimating the load of handed-over calls. Another advantage of DDBHP is that provides a solution for handovers in which the destination cell is not the next in the opposite direction of the satellite movement. The latter occasion is frequent if Earth movement and cells overlap are taken into account. Furthermore, DDBHP addresses the case of satellite handover (i.e. when the origin and the destination cells are in a different footprint) and provides a solution for cases that the destination satellite is in a different orbital plane.

This paper provides a theoretical analysis of the performance of DDBHP, leading to the formation of a Markov chain. The derivation of such a model is of essential importance to the actual operation of DDBHP because it associates traffic parameters and protocol variables (such as the threshold $t_{T H}$ defined in DDBHP), thereby allowing the choice of the desired system performance in terms of blocking and forced termination probabilities.

The rest of the paper is structured as follows. In Section II DDBHP is briefly presented for completeness of presentation and comments are made on its advantages, complexity and feasibility. Then, in Section III the proposed, for the analysis of DDBHP, Markov chain is presented and a methodology for determining $t_{T H}$ is proposed. Section IV discusses the simulation framework for evaluating the analytical formulas obtained and presents simulation as well as analytical results. Finally in Section V useful conclusions are drawn.

\section{The DopPler-BASEd HANDOVER PRIORITIZATION SCHEME}

The main objective of a successful handover procedure is to minimize and if possible eliminate the probability of forcing a call in progress into termination (forced termination probability - $P_{f}$ ). Although the minimization of $P_{f}$ is more important from the user point of view, $P_{b}$ (blocking probability) is also an important parameter for the network performance. Handover management involves always the tradeoff between $P_{f}$ and $P_{b}$. So far queuing and/or estimation of handover requests have been used to reserve resources. Most proposed techniques either fail to timely reserve resources and therefore suffer relatively high $P_{f}$ or reserve resource for prolonged periods of time thus resulting in relatively high $P_{b}$. In order to overcome the problem of protracted reservation of resources which increases $P_{b}$, DDBHP relies on Doppler effect monitoring to estimate not only the actual number of handover requests but also the actual time of occurrence. In this manner DDBHP reserves resources at the suitable time for minimizing both $P_{f}$ and $P_{b}$.

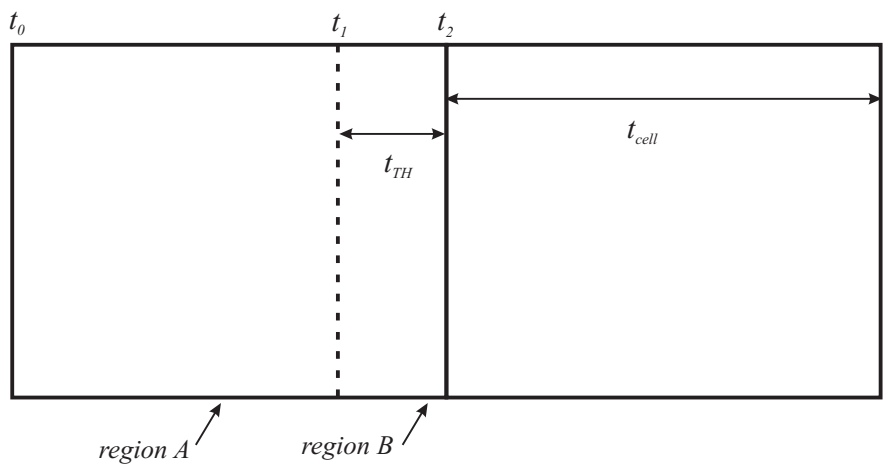

Fig. 1. Definition of time threshold in the DDBHP method

\section{A. The Basic Algorithm}

Let us consider the simplified case where a cell is approximated by the rectangle in Fig.1, an assumption widely used in the literature ([2],[3],[6]). DDBHP defines a time interval, called time threshold $\left(t_{T H}\right)$, prior to handover occurrence. The actual handover request is made at time $t_{1}$ instead of $t_{2}$. The system must complete resource reservation and achieve successful handover within $t_{T H}$ (it must be clear to the reader that $t_{T H}$ defines a region independent of the cells overlap area). It is clear that in order to implement such a procedure the mobile stations (MS) position must be known. For this reason DDBHP uses a position monitoring procedure based on Doppler effect that will be described in the next subsection. The criterion used to decide whether or not a new call will be admitted in the network, depends on the position of the MS at call setup:

- if the MS is located in region A an available channel only in the present cell is required

- otherwise available channels both in the present and in the next cell are required

For MSs admitted in the network the position monitoring mechanism provides the time they reach the $t_{T H}$ boundary. At this time a handover request is issued towards the appropriate satellite. In presence of available resources the request is satisfied (a channel is reserved), otherwise it is queued for time $t_{T H}$. If a channel is not found during this time, the call is forced into termination. If a call is terminated before the handover occurrence along with the serving channel, the reserved channel or the queued request are also cleared. It is clear that choosing properly $t_{T H}$ allows DDBHP to achieve different levels of $P_{f}$ or even eliminate it. The actual value of $P_{f}$ depends on $t_{T H}$, traffic and cell parameters as will be shown in Section III-C. The elimination of $P_{f}$ is achieved only when $t_{T H}=t_{\text {cell }}$. However, what is really of interest is that subject to the choice of $t_{T H}$, DDBHP can produce performances where $P_{f} \rightarrow 0$ (practical elimination of $P_{f}$ ). The tradeoff is that at the same time resources are more effectively managed since a user occupies two channels only for a period of $t_{T H}$ in the worst case.

\section{B. Position Monitoring}

The underlying hypothesis for implementing the described scheme is that the position of each MS is known to the 
serving satellite. DDBHP relies on estimating Doppler shift for each MS onboard the satellite to accomplish this task. The latter procedure is part of compensation techniques used for establishing communication between satellite and MS [9],[10] and therefore does not add any complexity. The advantage of measuring Doppler shift onboard the satellite is that location information regarding MSs is produced where is needed, thus avoiding the cost involved in its distribution. This is not the case when GPS is used to provide location information. Moreover, a GPS receiver must be incorporated in each MS, a solution not always feasible and cost effective. Therefore using Doppler shift is not only less complex but also more efficient than using any other location technique.

Monitoring of a MS's location consists of two phases. In the first one the serving satellite is able to derive the elevation angle of the communication at any time based on a single measurement of Doppler shift. This method has been used in literature ([11]) for other purposes. The second involves the calculation of the azimuth angle between the satellite direction and the MS by measuring Doppler shift at two different time instances. Consider the case in Fig.2. At time $t_{1}=0$ when a new call is admitted in the network and at $t_{2}=\Delta t$ the satellite measures the Doppler shift and therefore can derive the angular distances $\mathrm{AD}$ and $\mathrm{CD}$ :

$$
\begin{aligned}
& A D=\arccos \left(\frac{R_{E}}{R_{E}+h} \cdot \cos \hat{E}_{1}\right)-\hat{E}_{1} \\
& C D=\arccos \left(\frac{R_{E}}{R_{E}+h} \cdot \cos \hat{E}_{2}\right)-\hat{E}_{2}
\end{aligned}
$$

where $R_{E}$ is the Earth radius, $h$ the satellite altitude and $E_{1}$, $E_{2}$ are elevation angles at times $t_{1}$ and $t_{2}$ respectively. It can be shown that:

$$
\begin{aligned}
& \hat{E}_{1}=\arccos \left(-\frac{f_{D_{1}} \cdot \lambda}{2 \cdot \nu}\right) \\
& \hat{E}_{2}=\arccos \left(-\frac{f_{D_{2}} \cdot \lambda}{2 \cdot \nu}\right)
\end{aligned}
$$

where $f_{D_{1}}$ and $f_{D_{2}}$ are the measured Doppler shifts at $t_{1}$ and $t_{2}$ respectively, $\lambda$ is the transmission wavelength and $\nu$ the satellite velocity. The angular distance AC is calculated by:

$$
A C=\frac{2 \cdot \pi}{T_{s}} \cdot \Delta t
$$

where $T_{s}$ is the satellite period. By applying the law of cosines in the spherical triangle ADC the angle $\hat{\alpha}$ is derived:

$$
\hat{\alpha}=\arccos \left(\frac{\cos C D-\cos A C \cdot \cos A D}{\sin A C \cdot \sin A D}\right)
$$

By calculating the azimuth angle $\hat{\alpha}$, the satellite is able to derive the time at which a handover will occur as follows: In spherical triangle $\mathrm{ADB}$ angular distances $\mathrm{AD}$ and $\mathrm{BD}$ are known and related to $E_{1}$ and the minimum elevation angle $E$ respectively. By applying the law of sines we calculate $\hat{\gamma}$ :

$$
\hat{\gamma}=\arcsin \left(\frac{\sin B D}{\sin A D \cdot \sin \hat{\alpha}}\right)
$$

Angle $\hat{\beta}$ equals:

$$
\hat{\beta}=180^{0}-\hat{\alpha}-\hat{\gamma}
$$

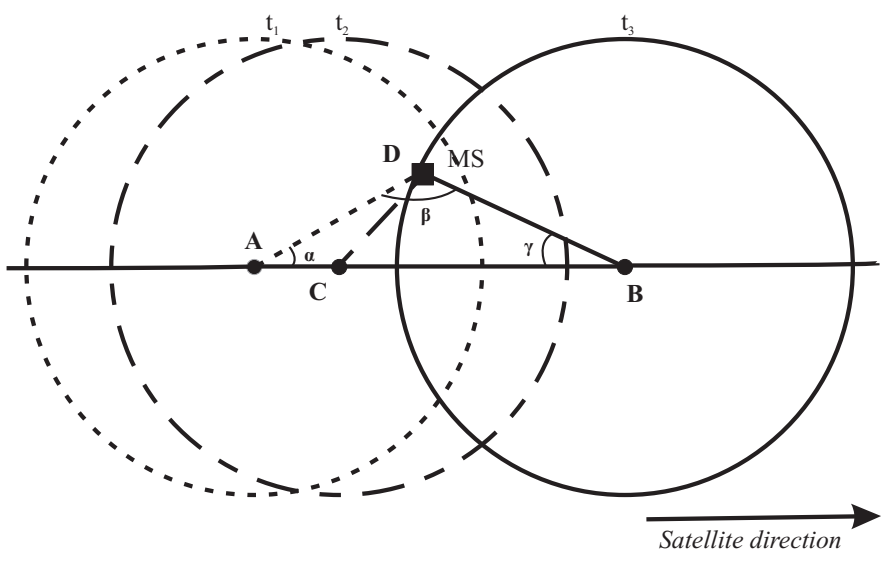

Fig. 2. The position monitoring method of DDBHP

Applying again the law of sines results in the angular distance $\mathrm{AB}$ :

$$
\hat{A B}=\omega_{F} \cdot t_{H}=\arcsin \left(\frac{\sin \hat{\beta} \cdot \sin \hat{\alpha}}{\sin B D}\right)
$$

with $\omega_{F}$ given by [11]:

$$
\omega_{F}=\omega_{s}-\omega_{E} \cdot \cos i \approx \omega_{s}
$$

where $\omega_{s}$ and $\omega_{E}$ are the angular velocities of the satellite and Earth respectively and $i$ the inclination of the orbital plane.

The accuracy for determining $t_{H}$ is subject to the error introduced by the measurement of $f_{D_{1}}$ and $f_{D_{2}}$. The estimation of the final error requires complex calculations using Eq. 1-9. However, compensation of the introduced error is fundamental to the proper operation of DDBHP because it affects $t_{H}-t_{T H}$ which indicates the time a handover request must be issued. To this end the system may follow two different approaches.

As will be shown in Section III-C false determination of $t_{H}$ results in failure to reach the desired levels of $P_{b}$ and $P_{f}$. The first method for compensating the error induced by measurements of Doppler shift involves successive adjustments on $t_{T H}$ in order to achieve the desired values for $P_{b}$ and $P_{f}$. The disadvantage of this method lies in the fact that for each MS a different error on $t_{T H}$ may be induced. On the other hand, the second approach compensates the introduced error for each MS. This is done by repeating the monitoring procedure proposed so far, more than once. Based on Eq. 3 and 4 it is clear that the induced error varies since elevation angle changes. The comparison of successive calculations of $t_{H}$ can produce its estimation. The cost for implementing this approach is minimal bearing in mind that Doppler shift monitoring is performed for other purposes.

\section{Determination of the Receiving Cell/Satellite}

Inherent in every method proposed so far in the literature is the assumption that in a handover the destination cell lies next to the serving cell in the opposite direction of the satellite movement. This is not always true especially when considering Earth rotation and overlap areas. DDBHP provides the possibility of identifying the destination cell. Let us consider the case in Fig. 3 where D is the location of the 


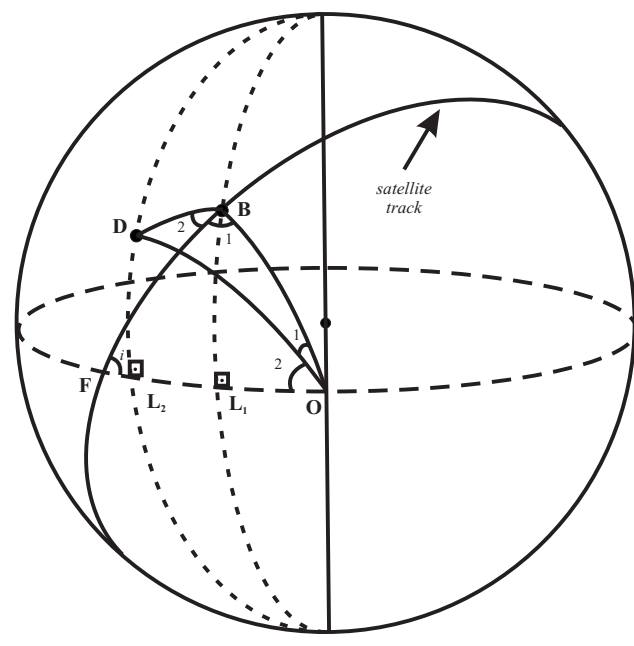

Fig. 3. The $3 \mathrm{~d}$ model for determining the receiving satellite

MS and B the sub-satellite point at a time that a handover occurs. By applying the law of sines in triangle FBO:

$$
\hat{O}=\arcsin \left(\frac{\sin F B \cdot \sin (i)}{\sin B O}\right)
$$

where the angular distance FB depends on the time elapsed since the satellite crossed the equatorial plane, $i$ is the orbit inclination and $\mathrm{BO}$ equals:

$$
B O=\arccos \left(\cos D L_{2} \cdot \cos L_{2} O\right)
$$

with $\mathrm{DL}_{2}$ and $\mathrm{L}_{2} \mathrm{O}$ being the latitude and longitude of the satellite respectively. Furthermore angle $\hat{B}_{1}$ equals:

$$
\hat{B}_{1}=180^{\circ}-i-\hat{O}
$$

Applying the law of cosines in triangle DBO leads to:

$$
\begin{aligned}
D O= & \arccos (\cos D B \cdot \cos B O \\
& +\sin D B \cdot \sin B O \cdot \cos \hat{B})
\end{aligned}
$$

where:

$$
D B=\arccos \left(\frac{R_{E}}{R_{E}+h} \cdot \cos \hat{E}\right)-\hat{E}
$$

and

$$
\hat{B}=\hat{B}_{1}+\hat{B}_{2}=\hat{B}_{1}+\hat{\gamma}
$$

Angle $\hat{\gamma}$ is given by Eq.7. By applying the law of sines in the same triangle we get:

$$
\hat{O}_{1}=\arcsin \left(\frac{\sin D B \cdot \sin \hat{B}}{\sin D O}\right)
$$

thus:

$$
\hat{O_{2}}=\hat{O}-\hat{O_{1}}
$$

Finally, applying the law of sines in the spherical triangle $\mathrm{DL}_{2} \mathrm{O}$ results in:

$$
\begin{gathered}
D L_{2}=\arcsin \left(\sin \hat{O}_{2} \cdot \sin D O\right) \\
L_{2} O=\arcsin \left(\sin \left(90^{0}-\hat{O}_{2}\right) \cdot \sin D O\right)
\end{gathered}
$$

By calculating the terminal location $\left(D L_{2}, L_{2} O\right)$ the serving satellite is able to derive the destination cell and make the reservation. Exact knowledge of the destination cell is important because the destination cell may be lateral to the serving one, instead of being the next in the opposite direction of satellite's movement. Moreover the serving satellite is able to decide if the destination cell belongs to a different satellite. If so, the serving satellite issues a reservation packet towards the destination satellite. The delivery of the reservation packet is managed through ISLs (inter-satellite links) and relies on the routing protocol. Different routing protocols can be used for this purpose [12]-[13]. Thus, obviously DDBHP supports cell as well as satellite handovers. The overhead involved in distributing reservation information in adjacent satellites can be considered low, bearing in mind that ISLs are usually implemented using high capacity optical links. Besides, in this way DDBHP averts monitoring of each MS in the overlap area by more than one satellite.

\section{ANALYTIC APPROACH FOR DDBHP}

It has been made clear so far that $t_{T H}$ defines the time in which the system must fulfill handover requests, thus straightforward affects performance in terms of $P_{f}$ and as a consequence of $P_{b}$. Therefore, determination of $t_{T H}$ has a central role in the implementation of DDBHP. A common methodology would be to perform extensive simulations for different constellations. The main disadvantage of this approach is its failure to dynamically follow changes on the system conditions, since only a fixed value of $t_{T H}$ is provided. On the other hand, a more convenient approach would be to derive an analytical formula describing the dependence of $t_{T H}$ on system and traffic specific parameters such as cell dimensions, available channels per cell, traffic intensity and traffic variation. Determination of $t_{T H}$ can be performed on the fly and for each satellite separately, in order to capture time and geographic variations of traffic and therefore to match system requirements more effectively.

This section aims at developing a Markov chain that would result in a formula associating performance metrics and $t_{T H}$, thus enabling the latter methodology.

\section{A. Basic Assumptions}

In order to derive a model for the analytical study of the proposed method, the concept of "street of coverage" [14],[2],[6] is adopted. It refers to a region covered by satellites in the same orbital plane and can be extended to represent a succession of cells ([6]). In this case cells are represented by rectangles as shown in Fig.1. The time to cross a cell is considered constant and given by [6]:

$$
t_{\text {cell }}=T_{s} \frac{l_{\text {cell }}}{2 \pi R_{E}}
$$

where $T_{s}$ is the satellite period, $l_{\text {cell }}$ the cell length and $R_{E}$ the Earth radius. The users are considered to be uniformly distributed within a cell and to travel in a direction opposite to the satellite direction with a velocity $\nu_{u}$. This velocity is equal to the satellite velocity and the same for each user. As proved in literature ([2],[6]) the probability that a user with a new call will produce the first handover is given by:

$$
P_{h_{1}}=\Gamma \cdot\left(1-e^{-\left(\frac{1}{\Gamma}\right)}\right)
$$




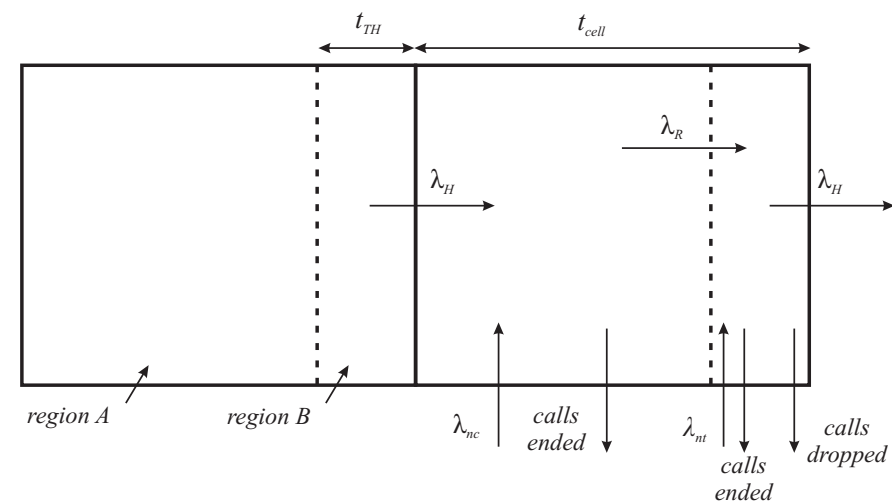

Fig. 4. Flux equilibrium for the DDBHP method

whereas the probability of generating a subsequent handover is:

$$
P_{h_{2}}=e^{-\left(\frac{1}{\Gamma}\right)}
$$

The parameter $\Gamma$ which is a metric of the system mobility is defined by:

$$
\Gamma=\frac{t_{\text {call }}}{t_{\text {cell }}}
$$

where $t_{\text {call }}$ is the mean call duration. In the following analysis we will concentrate on systems that use Fixed Channel Allocation (FCA). Each cell is considered to have a capacity of $\mathrm{C}$ channels.

The basic aim is to derive a Markov chain describing the state of each single cell. Since in our system exists the possibility of queuing, the model should be of type $M / M / C / K$ where $\mathrm{M}$ denotes the Markovian property for the processes of arriving and departing. $\mathrm{C}$ denotes the number of servers $(\mathrm{C}$ channels in our case) and $\mathrm{K}$ is the sum of servers and queue size. Keeping in mind Fig.4 it is clear that even if all users in one cell are handed over to the next one, the maximum queue size needed is $\mathrm{C}$. Therefore $\mathrm{K}$ equals to $2 \mathrm{C}$. The proposed model is based on some assumptions usually made in the literature ([2],[6],[3]):

- the Markovian property in the arrival process implies that an infinite number of users exists. Although this is impossible to implement in simulations a good approximation is achieved for a number of users $N_{\text {users }} \gg C$.

- the arrival process of handed-over calls is considered independent of the arrival process of new calls. Although this is not true, as stated in [6] it only results in overestimating blocking probability.

- the holding times in a cell for both new and handedover calls are considered exponentially distributed with different mean values [15]-[16].

- uniform traffic distribution is considered in each cell.

The validity of the described assumptions will be verified by the comparison of simulation and analytic results, made in Section IV.

\section{B. Traffic Components in a Cell}

The equilibrium state of a cell is depicted in Fig.4. In this, $\lambda_{H}$ is the rate of handed over calls, $\lambda_{R}$ the rate of handover requests and $\lambda_{n c}, \lambda_{n t}$ the rates of incoming calls in regions $\mathrm{A}$ and $\mathrm{B}$ respectively. The two later rates represent different processes that are subject to the different policies described in Section II-A. While users generated in region A require only a channel in the serving cell to be admitted into the network, the admittance of users in region B are subject to the existence of resources in the serving and the next cell. The equilibrium equations for one cell are:

$$
\begin{gathered}
\lambda_{R}=\lambda_{n c}\left(1-P_{b}\right) P_{h_{1}}^{\prime}+\lambda_{H} P_{h_{2}}^{\prime} \\
\lambda_{H}=\lambda_{n t}\left(1-P_{b}\right)^{2} P_{h_{1}}^{\prime \prime}+\lambda_{R}\left(1-P_{f}\right) P_{h_{2}}^{\prime \prime}
\end{gathered}
$$

therefore:

$$
\begin{aligned}
\lambda_{H}= & \frac{\lambda_{n t}\left(1-P_{b}\right)^{2} P_{h_{1}}^{\prime \prime}}{1-P_{h_{2}}\left(1-P_{f}\right)} \\
& +\frac{\lambda_{n c}\left(1-P_{b}\right)\left(1-P_{f}\right) P_{h_{1}}^{\prime} P_{h_{2}}^{\prime \prime}}{1-P_{h_{2}}\left(1-P_{f}\right)}
\end{aligned}
$$

In the equations above $P_{h_{1}}^{\prime}, P_{h_{1}}^{\prime \prime}, P_{h_{2}}^{\prime}$ and $P_{h_{2}}^{\prime \prime}$ are given by the following equations:

$$
\begin{aligned}
P_{h_{1}}^{\prime} & =\frac{t_{\text {call }}}{t_{\text {cell }}-t_{T H}}\left(1-e^{\frac{t_{\text {cell }}-t_{T H}}{t_{\text {call }}}}\right) \\
P_{h_{1}}^{\prime \prime} & =\frac{t_{\text {call }}}{t_{T H}}\left(1-e^{\frac{t_{T H}}{t_{\text {call }}}}\right) \\
P_{h_{2}}^{\prime} & =e^{\frac{t_{\text {cell }}-t_{T H}}{t_{\text {call }}}} \\
P_{h_{2}}^{\prime \prime} & =e^{\frac{t_{T H}}{t_{\text {call }}}}
\end{aligned}
$$

The first two represent the possibility that a newly generated call, in region $\mathrm{A}$ or $\mathrm{B}$ will cross the $t_{T H}$ and the cell boundaries respectively. The later two represent the possibility that a user will travel a fixed distance, equal to $t_{c e l l}-t_{T H}$ or $t_{T H}$ respectively, before its call ends. It is also clear that due to the different policies regarding users in regions $\mathrm{A}$ and $\mathrm{B}$, new call attempts are affected by the factors $\left(1-P_{b}\right)$ and $\left(1-P_{b}\right)^{2}$, where $P_{b}$ is the probability that there is no channel available in the cell. Accordingly the rate $\lambda_{R}$ is affected by the factor $\left(1-P_{f}\right)$, where $P_{f}$ is the probability that a user in queue will not finally find a channel within time $t_{\text {cell }}$.

\section{Resulting Markov Chain}

As explained in Section III-A each cell can be modeled as an $M / M / C / K$ system with $K=2 C$. The resulting chain can be seen in Fig.5. It is clear that states $[C+1,2 C]$ refer to the queue of each cell. The rate $\lambda_{t}$ which characterizes a jump from state $i$ to state $i+1, \forall i \in[0, C-1]$ is given by:

$$
\lambda_{t}=\lambda_{n c}+\lambda_{n t}\left(1-P_{b}\right)+\lambda_{n t}\left(1-P_{b}\right)+\lambda_{R}
$$

where $\lambda_{n c}$ represents the new calls in region A of the serving cell, each of the factors $\lambda_{n t}\left(1-P_{b}\right)$ the new calls generated in region $\mathrm{B}$ of the serving and the previous cell respectively and $\lambda_{R}$ the calls that request a handover. It must be noticed that rate $\lambda_{n t}$ is reduced by a factor $\left(1-P_{b}\right)$ because only calls that have secured a channel in the next cell can make a request for service in the current cell. It is clear that for jumps from state $i$ to state $i+1, \forall i \in[C, 2 C-1]$ the corresponding rate is $\lambda_{R}$. 


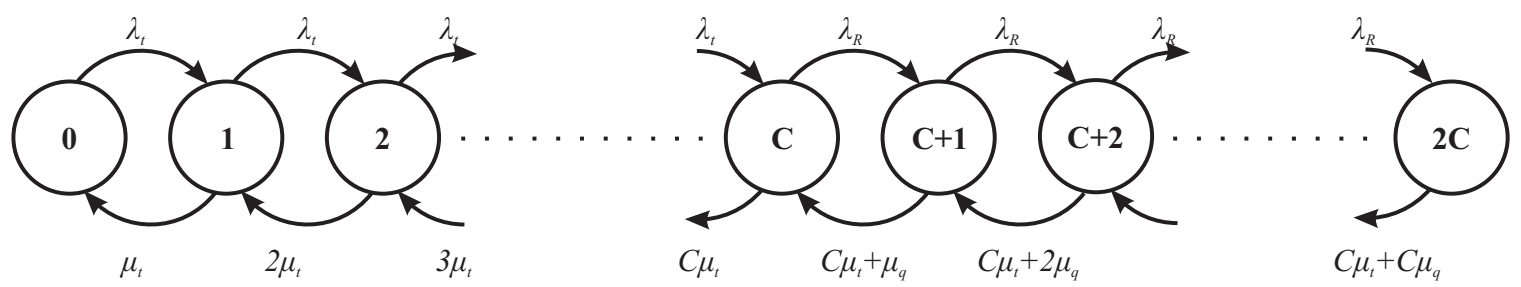

Fig. 5. The resulting Markov chain for DDBHP

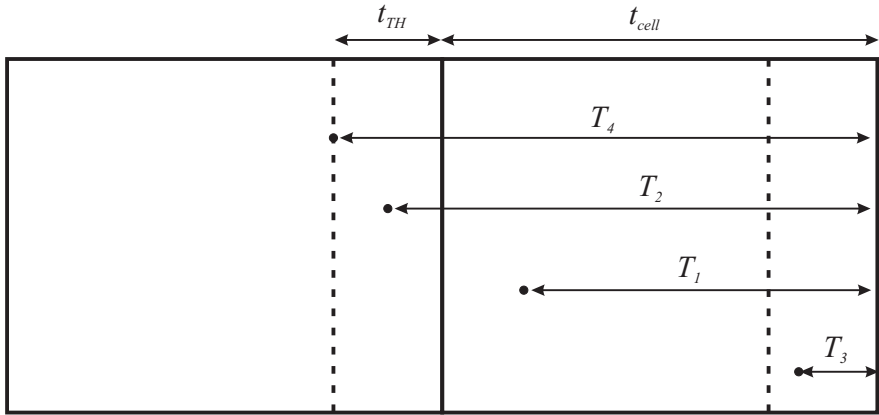

Fig. 6. Examples of holding times in a cell.

To determine the rate at which the system leaves state $i$ for state $i-1, \forall i \in[1, C]$ we need to calculate the mean holding time of a channel. Since a channel may be occupied by calls with different mean values the strategy, also adopted in literature ([6],[15]-[16]), will be to derive the composition of users in a cell and then average their mean holding times. Parameter A given by Eq.30 expresses the composition of users holding the channels of a cell.

$$
\begin{aligned}
A= & \lambda_{n c}\left(1-P_{b}\right)+\lambda_{n t}\left(1-P_{b}\right)^{2} \\
& +\lambda_{n t}\left(1-P_{b}\right)^{2}+\lambda_{R}\left(1-P_{f}\right)
\end{aligned}
$$

Parameter A consists of four components each of which represents a different case of user (Fig. 6). The first and second factor refer to new users generated in regions $\mathrm{A}$ and $\mathrm{B}$ of the cell respectively and have not yet performed any handover. The third factor represents users generated in region B of the previous cell that have not yet enter the cell under examination but hold a channel. Finally the fourth factor refers to users that have been handed over by the previous cell. The percentages for each of these components are given in Eq.31 while the mean holding time for each is given in Eq.32.

$$
\begin{aligned}
P_{1} & =\frac{\lambda_{n c}\left(1-P_{b}\right)}{A} \\
P_{2} & =\frac{\lambda_{n t}\left(1-P_{b}\right)^{2}}{A} \\
P_{3} & =\frac{\lambda_{R}\left(1-P_{f}\right)}{A} \\
E\left[T_{1}\right] & =t_{\text {call }}\left(1-P_{h_{1}}\right) \\
E\left[T_{2}\right] & =t_{\text {call }}\left(1-P_{h_{1}}^{\prime \prime} P_{h_{2}}\right) \\
E\left[T_{3}\right] & =t_{\text {call }}\left(1-P_{h_{1}}^{\prime \prime}\right) \\
E\left[T_{4}\right] & \left.=t_{\text {call }}\left(1-P_{h_{2}}^{\prime \prime} P_{h_{2}}\right)\right)
\end{aligned}
$$

Thus the average channel holding time in a cell, which is the reciprocal of rate $\mu_{t}$ is given by:

$$
\begin{aligned}
\frac{1}{\mu_{t}}= & t_{\text {call }}\left(P_{1}\left(1-P_{h_{1}}^{\prime}\right)+P_{2}\left(1-P_{h_{1}}^{\prime \prime} P_{h_{2}}^{\prime}\right)\right. \\
& \left.+P_{2}\left(1-P_{h_{1}}^{\prime \prime}\right)+P_{3}\left(1-P_{h_{2}}\right)\right)
\end{aligned}
$$

Finally for the transitions from state $i$ to state $i-1, \forall i \in[C+$ $1,2 C]$ there is an additional rate that represents the possibility that a call is ended while in the queue. Due to the memoryless property of the exponential distribution this rate is given by:

$$
\mu_{q}=1 / t_{\text {call }}
$$

Another assumption made is that $P_{f}$ is small and does not contribute to rate $\mu_{q}$. By solving the system of equations resulting from the equilibrium condition in each state of the chain the probability of state $n$ is derived:

$$
P_{n}= \begin{cases}\frac{\lambda_{t}^{n}}{n ! \mu_{t}^{n}} P_{0}, & \text { if } 0 \leq n<C \\ \frac{\lambda_{t}^{C} \lambda_{R}^{n-C}}{C ! \mu_{t} \prod_{j=1}^{n-C}\left[C \mu_{t}+j \mu_{q}\right]} P_{0}, & \text { otherwise. }\end{cases}
$$

where $P_{0}$ is calculated by:

$$
\begin{aligned}
P_{0}= & \left\{\sum_{n=0}^{C-1} \frac{\lambda_{t}^{n}}{n ! \mu_{t}^{n}}\right. \\
& \left.+\sum_{n=C}^{2 C} \frac{\lambda_{t}^{C} \lambda_{R}^{n-C}}{C ! \mu_{t} \prod_{j=1}^{n-C}\left[C \mu_{t}+j \mu_{q}\right]}\right\}^{-1}
\end{aligned}
$$

keeping in mind that $\sum_{n=0}^{2 C} P_{n}=1$. It is clear that the blocking probability $P_{b}$ is:

$$
P_{b}=\sum_{n=C}^{2 C} P_{n}
$$

To derive the probability $P_{f}$ that a call in the queue will not be served we can assume that rate $\mu_{q} \ll m_{t}$. $P_{f}$ is the probability that the queuing delay $W_{q}$ exceeds the limit of $t_{T H}$. This probability is given by:

$$
\begin{aligned}
P_{f} & =\operatorname{Pr}\left\{W_{q}>t_{T H}\right\} \\
& =\sum_{k=0}^{2 C} P_{k} \operatorname{Pr}\left\{W_{k+1}>t_{T H}\right\}
\end{aligned}
$$

which results in [17]:

$$
P_{f}=P_{b} e^{-C \mu_{t}\left(1-\frac{\lambda_{R}}{C \mu_{t}}\right) t_{T H}}
$$

It is clear that the aforementioned analysis is valid only when $P_{f}$ is small, an assumption easily made since as proved in [8] 
a suitable threshold can always be chosen to achieve small values of $P_{f}$.

Based on the analysis provided in this section and on Eq. 37 and $39, t_{T H}$ can be calculated for meeting the desired performance in terms of $P_{b}$ and $P_{f}$. Other involved parameters are constellation dependent and therefore considered known when facing a specific implementation of DDBHP. The rate of incoming traffic also affects $t_{T H}$. To this end, $t_{T H}$ may be set initially according to expected traffic rates and gradually adapt to the actual encountered traffic load. This process may be continuous, to give the ability of capturing date-time and geographical variations of traffic. Another advantage is that the system instead of using one system specific value for $t_{T H}$, may utilize different values for each satellite, each one specific to the traffic load offered to that satellite.

\section{Simulation And Analytic Results}

The purpose of this section is to evaluate the proposed analytical model. The comparison of DDBHP performance to other proposed methods [2],[6] has been made in [8], indicating an improved performance in terms of both blocking and forced termination probabilities. Results in [8] proved that DDBHP can guarantee the handover procedure, i.e. $P_{f}=0$, and at the same time minimize $P_{b}$. To validate the proposed analysis we used two LEO constellations resembling two proposed systems, Iridium [18] and Globalstar [19]. Because the proposed analysis involves recursive equations an iterative procedure was used to obtain $P_{b}$. For both systems we used $C=10$ channels per cell and traffic load ranging from 2 to 8 Erlang. We implemented a Poisson procedure for incoming traffic by using a number of users $N_{\text {users }}=1000 \gg C$ in both cases. The mean duration of generated calls was set to $t_{\text {call }}=180$ secs. Users were uniformly distributed in a cell. The other, system specific, parameters used are:

- Iridium system: $\gamma=2.38, t_{\text {cell }}=1.26 \mathrm{~min}$,

- Globalstar system: $\gamma=1.05, t_{\text {cell }}=2.85 \mathrm{~min}$

For the Iridium-like system Fig. 7 depicts $P_{b}$ with respect to the load offered to a cell when DDBHP is implemented. The convergence of analytical and simulation results proves that the proposed analysis successfully captures $t_{T H}$ dependence on the offered load. Thus, by using an iterative procedure each satellite of the constellation may determine $t_{T H}$ based on the monitored load. Fig. 7 proves that this methodology can be applied for a wide variation of the offered load. For high cell load the curves present a small divergence owed to the fact that under these conditions the assumption that $P_{f}$ is small, is not strong. In fact the divergence is intensified for smaller values of $t_{T H}$ because the available time for serving handover requests in the queue is smaller. In Fig. 8 the impact of $t_{T H}$ on the performance for a fixed cell load is illustrated. $P_{b}$ increases with $t_{T H}$ because more channels are locked by handover requests. Fig. 8 also proves the suitability of the proposed theoretical analysis in determining $t_{T H}$ based on the system desired performance.

To explore the way constellation specific characteristics (parameter $\gamma$ ) affect $t_{T H}$ we present in Fig. 9 and 10 the results for the Globalstar-like case. The performance of DDBHP

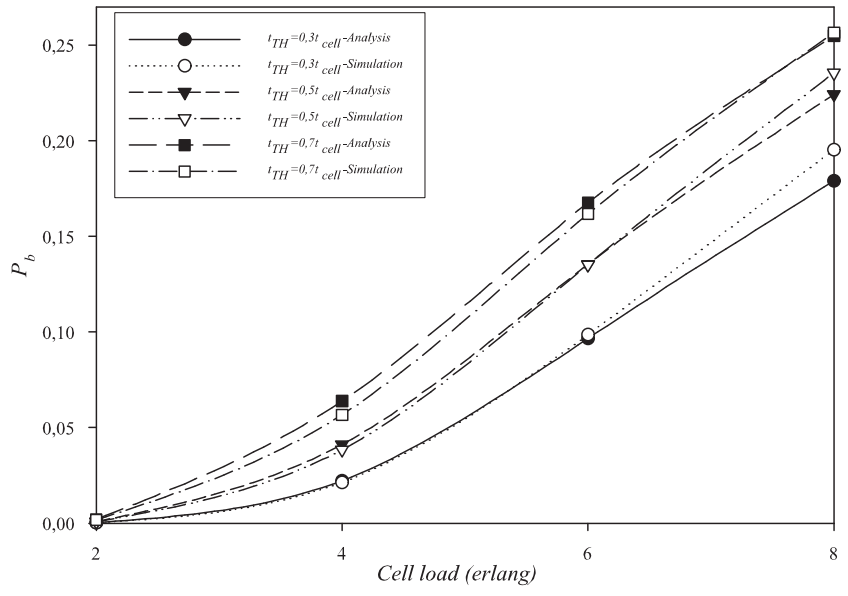

Fig. 7. Blocking probability versus cell load for the Iridium-like system

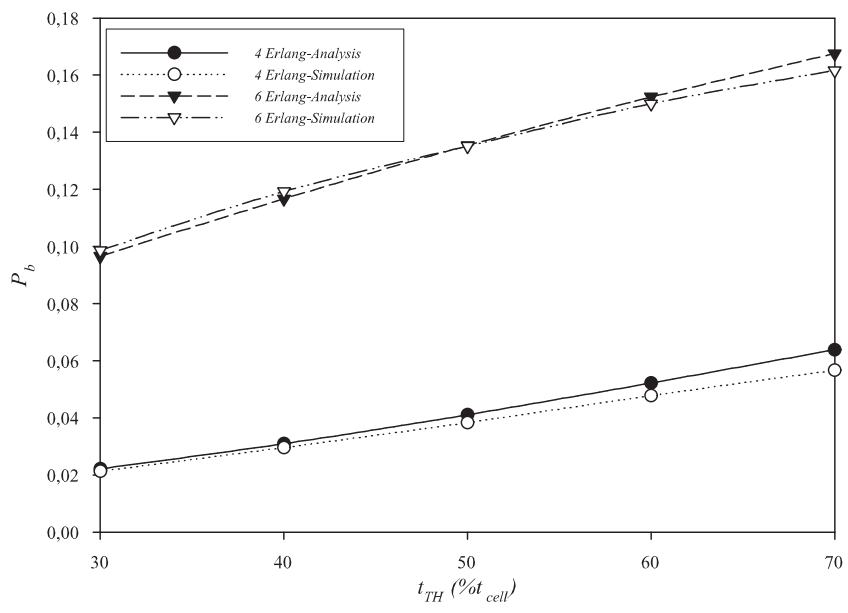

Fig. 8. Blocking probability versus threshlod for the Iridium-like system

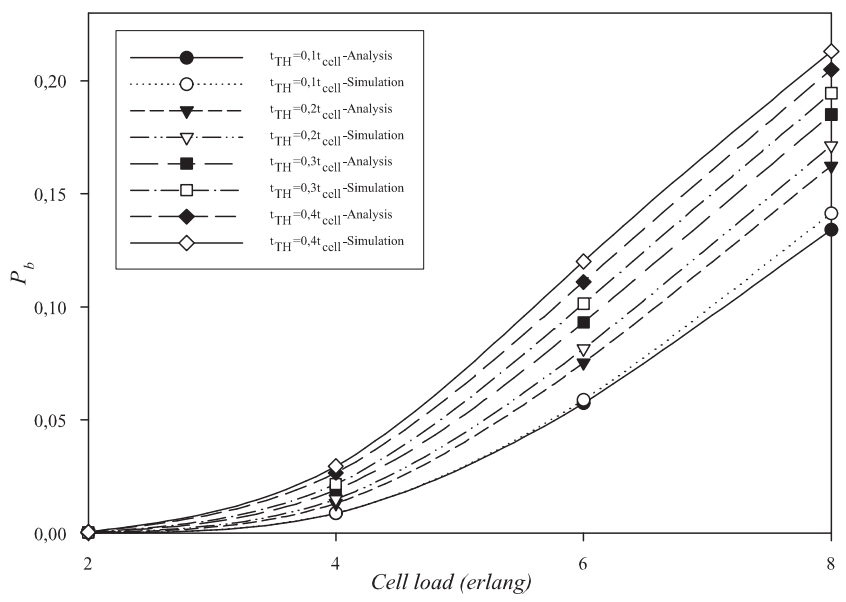

Fig. 9. Blocking probability versus cell load for the Globalstar-like system 


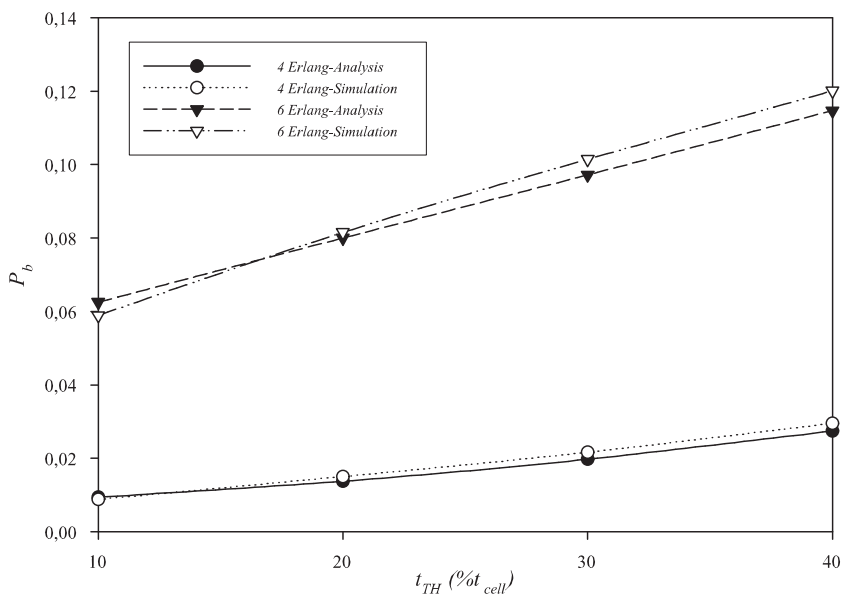

Fig. 10. Blocking probability versus threshold for the Globalstar-like system

is similar except that $t_{T H}$ is a smaller percentage of $t_{\text {cell }}$. This is because $t_{\text {cell }}$ is greater in this case. Conclusions made for the usefulness of the proposed analysis in the Iridium-like case, also stand for the Globalstar-like system, confirming the general applicability of the proposed analysis.

\section{CONCLUSION}

In this paper an analytical study of the method called Dynamic Doppler-based Handover Prioritization was presented. DDBHP manages handovers in LEO satellite fixed cell systems by taking advantage of Doppler effect to efficiently utilize system resources. Furthermore, DDBHP provides solution to handover cases that the destination cell is lateral to the serving cell and not the next in the opposite direction of the satellite movement, an issue that has never been addressed so far in the literature. The presented analysis resulted in the formation of a Markov model for determining DDBHP's key parameter, the time threshold $t_{T H}$. It was proved that the derived analytical formulas may be used to set $t_{T H}$ so as to meet a desired performance. At the same time, a methodology was provided for adapting $t_{T H}$ to time as well as geographic variations of offered load. Finally, issues concerning DDBHP's complexity and feasibility were addressed in this study. The presented theoretical analysis was validated for two typical LEO constellations through the comparison with extensive simulation results.

\section{REFERENCES}

[1] G. Maral and M. Bousquet, Satellite Communications Systems, 2nd ed. John Wiley, 1993.

[2] E. del Re, R. Fantacci, and G. Giambene, "Efficient dynamic channel allocation techniques with handover queuing for mobile satellite networks," IEEE J. Select. Areas Commun., vol. 13, no. 2, p. 397 405, February 1995.

[3] _ - "Different queuing policies for handover requests in low earth orbit mobile satellite systems," IEEE Trans. Veh. Technol., vol. 48, no. 2, pp. 448-458, 1999.

[4] D. Hong and S. Rappaport, "A traffic model and performance analysis for cellular mobile radio telephone systems with prioritized handoff procedures," IEEE Trans. Veh. Technol., vol. 35, no. 3, pp. 77-92, 1986.
[5] S. Tekinay and B. Jabbari, "Handover and channel assignment in mobile cellular networks," IEEE Commun. Mag., vol. 29, no. 11, pp. 42-46, 1991.

[6] G. Maral, J. Restrepo, E. del Re, R. Fantacci, and G. Giambene, "Performance analysis for a guaranteed handover service in an LEO constellation with satellite-fixed cell," IEEE Trans. Veh. Technol., vol. 47, no. 4, pp. 1200-1214, 1998.

[7] O. Ercetin, S. Krishnamurthy, S. Dao, and L. Tassiulas, "A predictive QoS routing scheme for broadband low earth orbit satellite networks," in Proc. IEEE International Symposium on Personal Indoor and Mobile Radio Communications (PIMRC' 00), vol. 2, London, UK, Sept 2000, pp. 1064-1074.

[8] E. Papapetrou and F.-N. Pavlidou, "QoS handover management in LEO/MEO satellite systems," Int. J. of Wireless Personal Communications, vol. 24, no. 2, pp. 189-204, January 2002, special Issue on Broadband Mobile Terrestrial-Satellite Integrated Systems.

[9] G. Povey and J. Talvitie, "Doppler compensation and code acquisition techniques for LEO satellite mobile radio communications," in Proc. IEE Fifth International Conference on Satellite Systems for Mobile Communications and Navigation (SSMCN' 96), London, UK, May 1996, pp. 16-19.

[10] S. Shin, K. Lim, K. Choi, and K. Kang, "Rain attenuation and doppler shift compensation for satellite communications," ETRI Journal, vol. 24 , no. 1, pp. 31-42, 2002.

[11] I. Ali, N. Al-Dhahir, and J. E. Hershey, "Doppler characterization for LEO satellites," IEEE Trans. Commun., vol. 46, no. 3, pp. 309-313, 1998.

[12] E. Papapetrou and F.-N. Pavlidou, "A proposal of optimal routing techniques for non-GEO satellite systems," IJWIN, vol. 8, no. 2, pp. 75-83, 2001.

[13] H. S. Chang, B. W. Kim, C. G. Lee, S. L. Min, Y. Choi, H. S. Yang, D. N. Kim, and C. S. Kim, "Performance comparison of static routing and dynamic routing in low earth orbit satellite networks," in Proc. IEEE Vehicular Technology Conference (VTC' 96), Atlanta, USA, April 1996, pp. 1240-1243.

[14] L. Rider, "Analytic design of satellite constellations for zonal earth coverage using inclined orbits," J. Astronautical Sciences, vol. 34, no. 1, pp. 31-64, 1986.

[15] D. K. Kim and D. K. Sung, "Handoff/resource managements based on PCVs and SVCs in broadband personal communication networks," in Proc. IEEE GLOBECOM' 96, London, UK, Nov 1996, pp. 18-22.

[16] K. W. Ross, Multiservice Loss Models for Broadband Telecommunication Networks. Springer-Verlag, 1995.

[17] F. Hillier and G. Lieberman, Introduction to Operations Research, 5th ed. McGraw Hill, 2002.

[18] (2003) The Iridium system. [Online]. Available: http://www.iridium.com

[19] (2003) The Globalstar system. [Online]. Available: http://www.globalstar.com

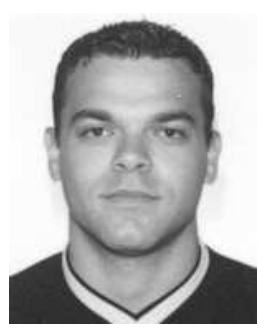

Dr. Evangelos Papapetrou holds a Diploma and a $\mathrm{PhD}$ in Electrical \& Computer Engineering from the Aristotle University of Thessaloniki, Greece. He is currently a visiting lecturer in the Computer Science Department at the University of Ioannina, Greece, where he is engaged in teaching and research on Mobile and Satellite Communications and Telecommunications Networks.

His research interests include traffic analysis and design of Satellite networks, Internet over Satellites, IP networking, routing in networks with periodic or stochastic varying topologies, MANETs and QoS in wireless mobile systems. He has served as a reviewer in several journals and Conferences relevant to mobile communications. In the past he has participated in Greek and European projects regarding satellite communications. He was also involved in COST Actions 253 and 272 and in many European projects undertaken by the Centre for Research and Technology Hellas (CERTH).

$\mathrm{He}$ is a Member of IEEE and the Joint VTS \& AES Greece Chapter and a member of Technical Chamber of Greece. 


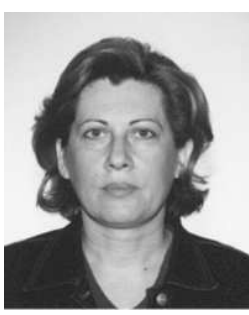

Dr. Fotin-Niovi Pavlidou holds a Diploma and a $\mathrm{PhD}$ in Telecommunications networks from the Aristotle University of Thessaloniki where she is currently engaged in teaching and research on Mobile Communications and Telecommunications Networks.

Her research interests include traffic analysis and design of networks, performance evaluation and QoS studies of mobile satellite communications and multimedia applications over Internet. She is a permanent reviewer in IEEE journals, she has served as Guest-Editor of Special issues on "Ad-Hoc Networks", "HAPs and applications", "PLC Systems and Applications" for International Journals like IJWIN, WPC etc. She is the author of a Chapter on Fixed Access Techniques (TDMA/FDMA) in the Wiley Encyclopedia on Telecommunications (Editor: Prof. John Proakis), and of many editions of COST Actions on "Satellite Systems", "Spread Spectrum Techniques" etc. She is the Delegate of Greece in the European COST Program on Telecommunications (1998-2004) and served as Chairperson for the COST262 Action "Spread Spectrum systems and techniques for wired and wireless Systems".

She is permanently included in the Program Committee of many IEEE conferences (PIMRC, GLOBECOM, VTC'2001, ISSSTA'2000) and she was the Chairperson of the IST Mobile Summit 2002, the annual conference of EU- Unit E4 in the field of Wireless Communications in Thessaloniki, June 16-20, 2002. She is involved in many European Projects (research or Education): Telematics Applications (INTERVUSE, ATTACH, etc), IST (ISMAEL,B-Bone, SatNEx, OPERA, etc), Tempus programs on Wireless Systems for Albania, Bulgaria, Poland.

She is a Senior Member of IEEE (Communications and Vehicular Technology Society), currently chairing the Joint VTS \& AES Greece Chapter. 